\title{
A Data Allocation Algorithm for Distributed Hypermedia Documents
}

\author{
Siu-Kai So, Ishfaq Ahmad, and Kamalakar Karlapalem \\ Department of Computer Science \\ The Hong Kong University of Science and Technology, Hong Kong \\ Email:\{kail, iahmad, kamal\}@ cs.ust.hk
}

\begin{abstract}
A major cost in executing queries in a distributed database system is the data transfer cost incurred in transferring relations (fragments) accessed by a query from different sites to the site where the query is initiated. The objective of a data allocation algorithm is to locate the fragments at different sites so as to minimize the total data transfer cost incurred in executing a set of queries. This is equivalent to minimizing the average query execution time, which is of primary importance in a wide class of distributed systems. The data allocation problem, however, is NP-complete, and thus requires fast heuristics to generate efficient solutions. The problem becomes more complex in the context of hypermedia documents (web pages), wherein these MDOs need to be synchronized during presentation to the end users. Since the basic problem of data allocation in distributed database systems is NP-complete, we need heuristics which can generate near-optimal MDO allocations. In this paper, we propose a navigational model to represent hypermedia documents and their access behavior from end users. We also formulate the problem by developing a base case cost model for response time and design an algorithm to find near-optimal solutions for allocating MDOs of the hypermedia documents while adhering to the synchronization requirements. We compare the algorithm solution with exhaustive solution over a set of experiments.
\end{abstract}

\section{Introduction}

Multimedia applications require, access, transfer and synchronization of multimedia data objects (MDOs) (such as, audio, video, and images) [1], [4]. The quality of services provided in presenting these MDOs to end-users has become an issue of paramount importance. End users have started expecting strict adherence to synchronization and response time constraints.

In order to manage and present large number of hypermedia documents (web pages) and their MDOs distributed hypermedia database systems are required. In fact, a set of web servers can be treated as a distributed hypermedia database system (DHDS). As the hypermedia documents may not be located at the end users sites, they need to be transferred across the communication network incurring delay (increasing response time) in presenting the MDOs of the hypermedia documents. Therefore, the allocation of the hypermedia documents and their MDOs govern the response time for the end-users. Moreover, as the MDOs in a hypermedia document need to be synchronized, the allocation should also adhere to these synchronization constraints.

A number of models for synchronization requirements (such as, HyTime [5], [9], OCPN [8], AOCPN [10], TPN [11] and $X O C P N$ [15]) have been proposed. A survey of $O C P N$ and its variants) is provided in [14]. The main problem with these models is that they do not provide any information about the expected number of times each state (representing $M D O$ or hypermedia document) is needed in a unit time interval. Without this information, the total response time in the DHDS cannot be estimated.

The contribution of this paper is the design and evaluation of a data allocation algorithm so as to optimize the response time for a set of end-users while adhering to the synchronization requirements of the MDOs presentation in DHDSs. We also propose a graph notion to represent navigation in the hypermedia systems and we introduce $O C P N$ modeling specification after that.

This paper is organized as follows: In the next section, we describe the hypermedia navigation model. In Section 3 we develop a cost model for the data allocation problem in $D H D S s$. In Section 4 we describe the proposed algorithm. In Section 5, we include the experimental results, and Section 6 summarizes the paper.

\section{Navigation Model for Hypermedia Documents}

We present a hypermedia system by a directed graph $D G(H, E)$ where $H=\left\{D_{1}, D_{2}, \ldots, D_{n}\right\}$ is the set of vertices, each $D_{p}$ representing a hypermedia document, and each directed edge from $D_{p}$ to $D_{p}$, is a link denoting access of document $D_{p}$, from document $D_{p}$. Therefore, a user can start browsing the documents from (say) document $D_{p}$ and then proceed to access document $D_{p^{\prime}}$, and so on. We have a label attached to each directed edge from $D_{p}$ to $D_{p}$, giving the probability of end users accessing document $D_{p}$, from document $D_{p}$. These probabilities are generated by gathering statistics (about document access, and browsing through logs of users browsing activity) about end-user behavior over a period of time. Further, since a user may end browsing after accessing any hypermedia document, the probabilities of out-going edges from a vertex do not add up to 1.0 , and the difference is the probability of ending the browsing at document $D_{p}$, and is shown by an edge connecting to the ground (see Figure 1). An $n \times n$ matrix navigation_prob is used to capture this information.

Example 1: Suppose we have four hypermedia documents $D_{1}-D_{4}$, Figure 1 shows the navigation model and the corresponding navigation_prob matrix.

From the above navigational model, we can calculate the probabilities of accessing a hypermedia document $D_{p}$, from document $D_{p}$. This is done by considering all possible paths to $D_{p}$, from document $D_{p}$ and calculating the probability of accessing $D_{p}$, from document $D_{p}$ for each path, and taking the maximum of all these probabilities. Note that we assume each document access and browsing from one document to another to 


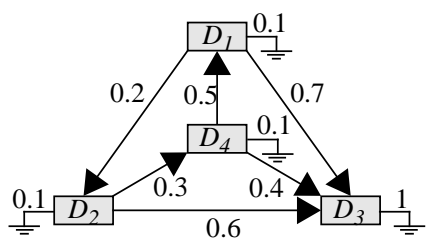

Figure 1: Probability model of navigational links between hypermedia documents.

be independent events. Therefore, for a path with $t$ edges from document $D_{p}$ to document $D_{p}$, the probability of this path is the product of $t$ probabilities for the edges. Since there can be potentially infinitely long paths, we limit the length of the path by limiting the value of the cumulative probability given by the path to be less than a parameter value $(b p l)$. Let $R$ be the $n \times n$ matrix, with each element $r_{j j}$, giving the cumulative long run probability of accessing document $D_{p}$, from document $D_{p}$.

Example 1 (Cont.): From the navigation model, we can construct a tree for each document representing the possible navigation path for each session starting from that document. These are given in Figure 2. We set the $b p l$ value to be 0.01 . Notice that we do not need to further expand a node if the document represented by that node is the same as that of the root. (This happens in the first tree in Figure 2). Therefore, if we start navigating the hypermedia system from document $D_{1}$, we have probability 0.2 that we browse document $D_{2}$. For document $D_{3}$, we have probability 0.7 if we follow the right path from $D_{l}$, but probability $0.2 \times 0.6=0.12$ if we follow the path $D_{1} \rightarrow D_{2} \rightarrow D_{3}$. In this case, we use the greater probability to represent the long run probability of browsing $D_{3}$ from $D_{1}$ as 0.7 . Similarly other cumulative probability values are calculated.

Therefore, the matrix $R$ is

$\begin{aligned} & D 1 \\ & D 2 \\ & D 3 \\ & D 4\end{aligned}\left[\begin{array}{cccc}1 & 0.2 & 0.7 & 0.06 \\ 0.15 & 1 & 0.6 & 0.3 \\ 0 & 0 & 1 & 0 \\ 0.5 & 0.1 & 0.4 & 1\end{array}\right]$.
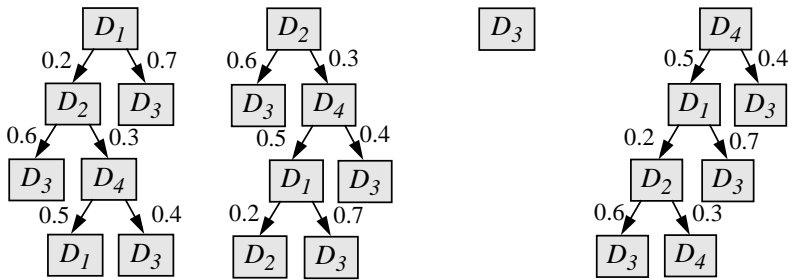

Figure 2: Navigation path starting from each hypermedia document (bpl is set as 0.01).

We use the $O$ bject $C$ omposition Petri Nets (OCPNs) [8] for modeling the synchronization constraints among the MDOs in a hypermedia document. OCPN simplifies the Petri nets by restricting the number of outgoing edges from each Transition to two and it enhances them by introducing duration and address (locating the MDO) for each Place. This enhancement makes $O C P N$ suitable for modeling synchronization constrains among
MDOs of hypermedia documents. We can transverse a Transition (called as firing) if all Places pointing to this Transition have a token and are in an unlocked state. When the Transition fires, the Places that the Transition is pointing to will become active (a token is added to these Places) and locked. Places will become unlocked when their durations have expired. All OCPN models can be mapped to a corresponding HyTime model [2]. In Figure 3 , the following synchronization constraint is represented in OCPN1: MDO $A$ has to be shown exactly 40 units of time after the start of browsing the hypermedia document, and in parallel after 55 units of time MDO $B$ must be shown. The $O C P N$ specifications of hypermedia documents $D_{1}$ to $D_{4}$ are shown in Figure 3.
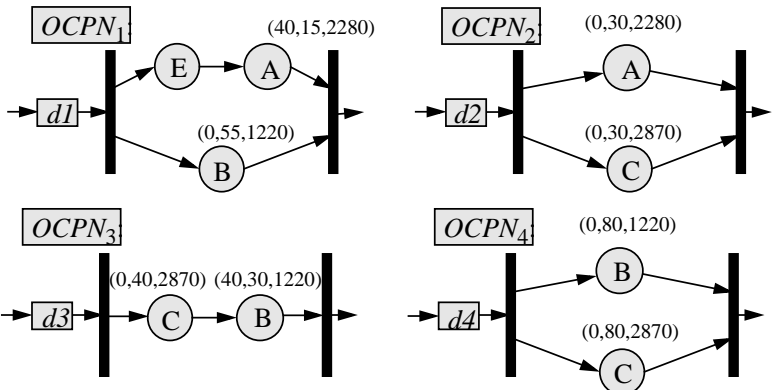

Figure 3: The $O C P N$ specification of each hypermedia document; the tuple is [start time, duration, media size in kilobytes].

\section{Cost Model of Data Allocation Scheme}

Table 1 lists a number of notations used throughout this paper.

Table 1: Symbols and their meanings.

\begin{tabular}{|c|c|}
\hline Symbol & Meaning \\
\hline$S_{i}$ & The $i$ th site \\
\hline$D_{j}$ & The $j$ th hypermedia document \\
\hline$O_{k}$ & The $k$ th $M D O$ \\
\hline $\mathrm{m}$ & The number of sites in the network \\
\hline $\mathrm{n}$ & The number of hypermedia documents in the database system \\
\hline $\mathrm{k}$ & The number of $M D O \mathrm{~s}$ in the database system \\
\hline$P$ & The user navigation pattern matrix of site $i$ \\
\hline$p_{j j^{\prime}}^{i}$ & $\begin{array}{l}\text { The probability of using document } j^{\prime} \text { as initial browsing document if the } \\
\text { initial browsing document is } j \text { in the previous navigation session at site } i\end{array}$ \\
\hline B & The navigation initial document frequencies matrix \\
\hline$b_{i j}$ & The frequency of using the $j$ th document as initial point at the $i$ th site \\
\hline $\mathrm{C}$ & The transmission speed matrix of the network \\
\hline$\overline{c_{i i^{\prime}}}$ & The transmission speed from site $i$ to site $i^{\prime}$ \\
\hline A & The access frequencies matrix \\
\hline$a_{i j}$ & The access frequency of document $j$ from site $i$ \\
\hline 1 & The allocation limit vector of the sites \\
\hline$l_{i}$ & The allocation limit of site $i$ \\
\hline $\mathrm{R}$ & The hypermedia document dependency matrix \\
\hline$r_{i j^{\prime}}$ & The probability of retrieving document $j^{\prime}$ if browsing initial document is $j$ \\
\hline$O C P N_{j}$ & The $O C P N$ specification of document $j$ \\
\hline $\mathrm{U}$ & The use matrix \\
\hline$u_{i k}$ & The boolean value of whether document $j$ uses $M D O k$ \\
\hline $\mathrm{dur}_{j k}$ & The presentation duration of $M D O k$ in document $j$ \\
\hline start $_{j k}$ & The presentation starting time of $M D O k$ in document $j$ \\
\hline $\operatorname{size}_{k}$ & The size of the $k$ th $M D O$ \\
\hline bpl & The browsing probability limit. \\
\hline$e t_{j}$ & The expected number of times document $j$ will be retrieved \\
\hline $\mathrm{D}$ & The delay matrix \\
\hline$d_{i j}$ & The delay of presentation starting time of document $j$ at site $i$ \\
\hline $\mathrm{t}$ & The total delay \\
\hline
\end{tabular}

In order to reduce response time for the end-users browsing 
activities, we need to develop a cost model for calculating the total response time observed. This response time depends on the location of the MDOs and the location of the end-user. Further, it depends on the synchronization constraints among the MDOs of the hypermedia document browsed. The hypermedia document navigational model presented in Section 2 is used to estimate the number of accesses (times browsed) to each MDO from each site. This gives us information regarding affinity between the MDOs and the sites of the distributed environment. Typically, one would assign a MDO to a site which accesses it most. But this may incur large delay for other sites which need to access this MDO. Further, synchronization constraints may impose additional delays in transferring the MDO to the end-user site. This is done when two streams of MDOs need to simultaneously finish their presentation, and one of them is for shorter duration than the other. Since we are buffering the MDOs at the user sites before the start of the presentation, the MDO allocation problem needs to minimize this additional delay that is incurred because of the synchronization constraints. We also take into consideration limited buffer space constraint at end-users site and user interaction during MDO presentation.

\subsection{The Cost Function}

Suppose there are $m$ sites in the distributed hypermedia database system. Let $S_{i}$ be the name of site $i$ where $1 \leq i \leq m$. These $m$ sites are connected by a communication network. A communication link between two sites $S_{i}$ and $S_{i^{\prime}}$ will have a positive integer $c_{i i^{\prime}}$ associated with it giving the transmission speed from site $i$ to site $i^{\prime}$. Notice that these values are depended on the routing scheme of the network. If fixed routing is used, we can have the exact values. However, if dynamic routing is in used, we can only obtain the expected values. Let there be $n$ hypermedia documents, called $\left\{D_{1}, D_{2}, \ldots, D_{n}\right\}$ accessing $k$ $M D O$ s, named $\left\{O_{1}, O_{2}, \ldots, O_{k}\right\}$.

From the navigation model, we can construct $n$ trees representing the navigation path of the session starting from each document. As in Section 2, we must limit the level we will use for our cost model by a threshold value $b p l$, say 0.001 . These trees will give us some information about the probability $r_{j j^{\prime}}$ of retrieving the document $j^{\prime}$ if we start navigating from the $j$ th document.

For each site, we use an irreducible continuous-timed Markov process [13] to model the user behavior in initial browsing document (i.e., the document first browsed) as a stationary regular transition probability matrix, $P^{i}, 1 \leq i<m$. These processes will converge in the long run and from these long run behaviors, we can estimate the probability of browsing each document from each site as the initial browsing document. These Markov chains will have $n+1$ states representing the probabilities of using each of the documents as the initial browsing document (n states), and probability of not browsing any of the documents $((n+1)$ th state). After analyzing the long run behavior of the Markov chain at each site, we will have the probabilities of using each document as initial browsing document and of not browsing at each site. As there is no delay when the user does not browse, we can eliminate the probability of not browsing. Normalize the remaining probabilities and multiply them by a constant $T$, we have the expected frequencies of initial document out of $T$ browsing sessions. The resultant information is represented by an $m \times n$ matrix $B$.

We multiply this matrix to the $n \times n$ matrix $R$ obtained from the hypermedia document trees to generate an $m \times n$ matrix $A$ with entries $a_{i j}$ giving the expected number of times $S_{i}$ needs to retrieve the $M D O$ s in $D_{j}$. Further, we need the starting time, duration, size, and presentation rate of each $M D O$ in each hypermedia document. This information can be obtained from the OCPN specification of MDOs in a hypermedia document.

A box will be added at the beginning of each $O C P N$ which represents the delay in starting the presentation of the hypermedia document so as to adhere to the synchronization requirements. The duration of this delay box is related to the browsing site and the sites where the MDOs in the document are allocated. Thus, we use $d_{i j}$ to represent the duration of the delay box when site $S_{i}$ accesses document $D_{j}$.

From the $O C P N$ representations, we have the starting time start $_{j k}$ and duration $d u r_{j k}$ of each $M D O O_{k}$ in each document $D_{j}$. In addition, the $n \times l$ usage matrix $U$ is generated from the $O C P N$ specifications. If document $D_{j}$ uses $M D O O_{k}$, then set $u_{j k}$ to 1 , otherwise, set $u_{j k}$ to 0 . Then, by multiplying $A$ by $U$, we can estimate the access frequencies of each $M D O$ from each site. Let size $_{k}$ be the size of $M D O O_{k}$.

With this information, we can calculate $d_{i j}$ by,

$$
d_{i j}=\max _{\forall k, u_{j k}=1}\left(\frac{\text { size }_{k}}{c_{\text {site }(k) \cdot i}}-d u r_{j k}-\operatorname{start}_{j k}\right)
$$

where $\operatorname{site}(k)$ represents the site where $O_{k}$ is allocated.

We can calculate the values of all $d_{i j}, 1 \leq i \leq m, 1 \leq j \leq n$, by using the above formula. This formula means that the delay is equal to the maximum value of (transmission duration presentation duration - presentation starting time) for each $M D O$ in the document. When this value is negative, implying that the transmission time is shorter than the presentation time, we can start presenting the MDOs in the hypermedia document as soon as the MDOs arrive at the end-user site. When this value is positive, we know that we must delay the presentation, otherwise the MDOs presentation will end before the synchronization time, and hence will not adhere to the synchronization constraints.

Therefore, we have the cost function,

$$
t=\sum_{i \in S} \sum_{j \in D} d_{i j} \cdot a_{i j} \quad \text { EQ } 3.2
$$

By minimizing this value through the change of the function $\operatorname{site}(k)$, we obtain the data allocation scheme that is optimal (the (delay incurred) response time is minimal), while adhering to the synchronization constraints.

\subsection{User Interactions and Buffer Space Constraints}

The model presented above does not consider user interactions and buffer space constraints. It assumes that the user does not interrupt the presentation and the size of the local storage facility is large enough for storing any one of the hypermedia documents in the hypermedia database system. We only need to be concerned about the probabilities of each user interaction; therefore, we will not introduce these models here. By including user interactions and buffer space constraints, there can be four different cases for hypermedia document allocation 
problem given below.

\subsubsection{No user interaction and unlimited buffer space}

This is essentially the best scenario, because we can retrieve all $M D O$ s in a hypermedia document at the beginning since there is no storage limitation. As there is no user interaction, the data can be discarded immediately after use. The cost function for the response time for each hypermedia document, as presented in Section 3.1, is the maximum of the delays of the embedded $M D O$ s for satisfying the synchronization requirements.

\subsubsection{User interaction and unlimited buffer space}

By including user interactions, some of the MDOs in a hypermedia document may need to be presented multiple times (e.g. play in reverse or stop and resume later). However, as there is unlimited buffer space, the system can store all MDOs of a hypermedia document once they are retrieved. Therefore, the delay for handling the user interactions is some local processing time that is irrelevant to the data allocation of the $M D O$ s. The cost function is thus same as that in the Section 3.1.

\subsubsection{No user interaction and limited buffer space}

In this scenario, the system can not use the retrieving all the MDOs in advance strategy. Instead, the system must retrieve the $M D O s$ only when it needs to present these $M D O$ s. Therefore, every synchronization point in the hypermedia document may cause some delay and the cost function in such situation is the summation of these delays. Indeed, the model presented in Section 3.1 can be generalized to deal with this scenario.

First, we need to decompose each document into component sub-documents. From the $O C P N$ specifications, we have the states representing the $M D O$ s in each document. Denote this set of states as $S$ and for $\forall s, s \in S$, we can get the starting time and ending time of the state (i.e., presentation of the corresponding $M D O$ ) from the $O C P N$ specifications. Then, we can decompose the document by composing all $M D O$ s starting at the same time into a sub-document (so if there are $h M D O s$, there will be $h$ subdocuments in maximum).

\subsubsection{User interaction and limited buffer space}

If we know the expected number of times each sub-document will be presented in each hypermedia document, we can calculate the expected response time of each document in each site. It is just the weighted sum of the delays of the sub-documents in the document. To calculate the expected number of times each document is needed, we must know the probabilities of relevant user interactions (such as reverse playing, and fast forward). Once we have these probabilities, we can calculate the expected number of time each document is presented by employing the first step analysis method [13]. Note that these probabilities can be generating after observing user interaction over a period of time.

For example, suppose the relevant probability of an $M D O k$ in a document $j$ is $i p_{j k}$. Assume that the expected number of time this $M D O$ is needed is $m d o \_e t_{j k}$. Then, we have ${ }^{\dagger}$,

$$
\begin{aligned}
m d o \_e t_{j k} & =1+i p_{j k} \times m d o \_e t_{j k}, \\
m d o \_e t_{j k} & =1-i p_{j k}{ }^{-1} \quad \text { EQ } 3.3
\end{aligned}
$$

$\dagger$. or $m d o \_e t_{j k}=1+i p_{j k}+i p_{j k}^{2}+\ldots=\left(1-i p_{j k}\right)^{-1}$
Similarly, we can estimate the expected number of times other $M D O$ s composing this document are needed. Then, the expected number of times this document is needed is just the maximum of these values. Denote this value as $e t_{j}$ for document $D_{j}$, we have,

$$
e t_{j}=\max \left(m d o \_e t_{j k}\right), \text { for } \forall k, u_{j k}=1
$$

And the delays $d_{i j}$ will become,

$$
d_{i j}=\left(\max \left(\frac{\text { size }_{k}}{c_{\text {site }(k) \cdot i}}-d u r_{j k}-\operatorname{start}_{j k}\right)\right) \cdot e t_{j}, \text { for } \forall k, u_{j k}=1 \text {. }
$$

Example 1 (Cont.): Assume that the hypermedia database system for storing the $M D O$ s is distributed in a network with 3 sites.

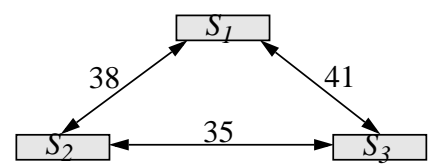

Figure 4: The transmission speed between the sites in Kilobytes per second.

In Figure 4, the transmission speed between the 3 sites are given. These values can be represented as an $m \times m$ matrix $C$, with entry $c_{i i^{\prime}}$ representing the transmission speed from $S_{i}$ to $S_{i^{\prime}}$.

$\begin{array}{cccc}S 1 & S 2 & S 3 \\ S 1 & {\left[\begin{array}{ccc}0 & 38 & 41 \\ 38 & 0 & 35 \\ 41 & 35 & 0\end{array}\right] .}\end{array}$

Suppose after the analyses of the long run behavior of the Markov chain in each site, the expected starting document frequencies out of 900 browsing sessions, matrix $B$ is,

$$
\begin{aligned}
& \begin{array}{llll}
D 1 & D 2 & D 3 & D 4
\end{array} \\
& S 1 \quad\left[\begin{array}{llll}
100 & 200 & 300 & 200
\end{array}\right] \\
& \begin{array}{lllll}
S 2 & 225 & 450 & 225 & 0
\end{array} \\
& S 3\left[\begin{array}{llll}
300 & 100 & 100 & 400
\end{array}\right] \text {. }
\end{aligned}
$$

Then the matrix $A(B \times R)$ is,

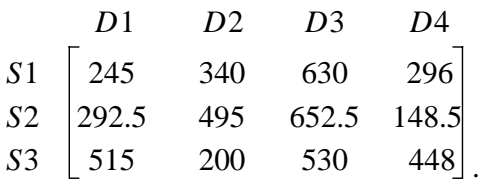

In this example, there are $3 \mathrm{MDO}$ s, namely $\mathrm{A}, \mathrm{B}$ and $\mathrm{C}(\mathrm{E}$ is a delay state, so there is no associative actual $M D O$ ). If we allocate A at Site 2, B at Site 3 and C at site 1, then $d_{11}$ is equal to,

$$
d_{11}=\max \left(\left(\frac{2280}{38}-15-40\right),\left(\frac{1220}{41}-55-0\right)\right)=5 \text {. }
$$

Similarly, we can calculate the values of all $d_{i j}, 1 \leq i \leq 3,1 \leq j \leq 3$ when we have the $M D O$ allocation scheme. And the total response time delay will be,

$$
t=11430+4573.25+29130=86291.25 .
$$

Suppose we add user interactions and worst case buffer space constraints to this hypermedia database system. After adding the 
probability of relevant user interruption to the $M D O$, the augmented $O C P N$ of $D_{1}$ is shown in Figure 5.

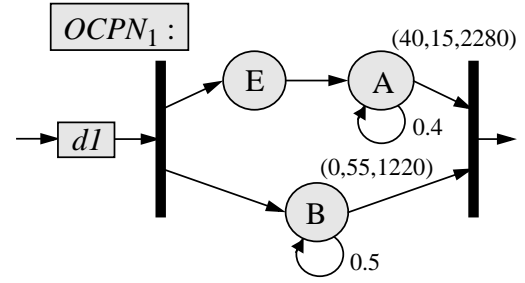

Figure 5: The augmented OCPN by including user-interaction.

Thus, the expected number of times $M D O A$ is needed when document $D_{1}$ is retrieved is,

$$
\begin{gathered}
m d o \_e t_{1 A}=1+0.4 \times m d o \_e t_{1 A}, \\
m d o \_e t_{1 A}=\frac{1}{1-0.4}=1.667 .
\end{gathered}
$$

Similarly, the expected number of times $M D O B$ is needed is,

$$
\begin{gathered}
\text { mdo_et } t_{1 B}=1+0.5 \times \text { mdo_et } t_{1 B}, \\
\text { mdo_et } t_{1 B}=\frac{1}{1-0.5}=2 .
\end{gathered}
$$

Notice that when we need $B$ again, $A$ is also needed. Thus, $e t_{1}=\max (1.667,2)=2$.

Since we have worst case buffer space constraints, so the delay $d_{11}$ will become

$$
d_{11}=\left(\max \left(\left(\frac{2280}{38}-15-40\right),\left(\frac{1220}{41}-55-0\right)\right)\right) \times 2=10 .
$$

\section{The Data Allocation Algorithm}

As mentioned above, the data allocation problem in its simple form is NP-complete [3] and the problem discussed here is more complex than the simple case; there are $k^{m}$ different allocation schemes for a system with $m$ sites and $k M D O$ s, implying that an exhaustive search would require $O\left(k^{m}\right)$ in the worst case to find the optimal solution. Therefore, we need heuristic algorithms to solve the problem.

We have developed an algorithm based on the Hill-Climbing technique to find a near optimal solution. The data allocation problem solution consists of the following two steps:

1) Find an initial solution.

2) Iteratively improve the initial data allocation by using the hill climbing heuristic until no further reduction in total response time can be achieved. This is done by applying some operations on the initial allocation scheme. Since there are finite number of allocation schemes, the heuristic algorithm will complete its execution.

For step one, one possibility is to obtain the initial solution by allocating the $M D O$ s to the sites randomly. However, a better initial solution can be generated by allocating an $M D O$ to the site which retrieves it most frequently (this information can be obtained from the matrix $A \times U)$. If that site is already saturated, we allocate the $M D O$ to the next site that needs it the most. We call this method the $M D O$ site affinity algorithm.

In the second step, we apply some operations on the initial solution to reduce the total response time. Two types of operations are defined, namely migrate (move $M D O$ s from its currently allocated site to another site) and swap (swap the locations of one set of $M D O \mathrm{~s}$ with the locations of another set of $M D O$ s). These operations are iteratively applied until no more reduction is observed in the total response time.

migrate $\left(O_{j}, S_{i}\right)$ : move $M D O O_{j}$ to $S_{i}$. This operation can be applied to each $M D O$, and an $M D O$ can be moved to any one of the $m-1$ sites at which it is not located. Therefore, there can be a maximum of $k(m-1)$ migrate operations that can be applied during each iteration.

$\operatorname{swap}\left(O_{x}, O_{x^{\prime}}\right)$ : swap the location of $M D O O_{x}$ with the location of $M D O O_{x^{\prime}}$. This operation can be applied to each distinct pair of $M D O$ s. Therefore, there can be a maximum of $k(k-1) / 2$ swap operations that can be applied during each iteration. Although this operation is equivalent to two migrate operations, it is necessary as some of the sites may be already saturated such that we cannot migrate $M D O$ to it any more.

\section{Experimental Results}

In this section, we present the experimental results for the data allocation algorithm described in Section 4. Since for $k$ $M D O$ s and $m$ sites there are $k^{m}$ allocation schemes for exhaustive search algorithm, the problem sizes of the experiments we conducted were limited. We conducted 25 experiments with number of $M D O$ s ranging from 4 to 8 , and number of sites ranging from 4 to 8 . Each experiment consisted of 100 allocation problems with the number of sites and the number of $M D O \mathrm{~s}$ fixed. Each allocation problem had between 4 and 16 documents, and each document used a subset of the MDOs with its own temporal constraints on them. The communication network, the $M D O$ sizes, the link costs, and the temporal constraints between $M D O$ s in each document were randomly generated from a uniform distribution. The data allocation algorithm described above was tested for every case and statistics was collected.

In Table 2, for each of the experiments conducted in a column-wise fashion, we list the following: i) the number of sites, ii) the number of $M D O \mathrm{~s}$, iii) the number of problems for which optimal solutions are generated, iv) the average deviation in percentage of near optimal solutions from optimal solution when optimal solution was not reached. The number of optimal solutions can reflect how good the algorithm is; whereas the average deviation shows how bad the algorithm performs when it cannot generate optimal solution.

Table 2: Experimental results of the proposed algorithm.

\begin{tabular}{|c|c||c|c|}
\hline $\begin{array}{l}\text { No. of } \\
\text { Sites }\end{array}$ & $\begin{array}{l}\text { No. of } \\
\text { MDOs }\end{array}$ & $\begin{array}{l}\text { No. of Opt. } \\
\text { Sol. (H) }\end{array}$ & $\begin{array}{l}\text { Aver. \% } \\
\text { Deviat. (H) }\end{array}$ \\
\hline \hline 4 & 4 & 93 & 15.4163 \\
\hline 4 & 8 & 81 & 5.3714 \\
\hline 5 & 4 & 92 & 19.3567 \\
\hline 5 & 8 & 83 & 11.5364 \\
\hline 6 & 4 & 97 & 5.2258 \\
\hline 6 & 8 & 82 & 11.6584 \\
\hline 7 & 4 & 88 & 3.0582 \\
\hline 7 & 8 & 77 & 3.9969 \\
\hline 8 & 4 & 88 & 3.8620 \\
\hline 8 & 8 & 72 & 9.7750 \\
\hline
\end{tabular}

From Table 2, we note that the proposed algorithm generated 
optimal solutions for a large number of problems -2122 cases out of a total of 2500 cases, corresponding to about $85 \%$ of the test cases. Most of the non-optimal solutions are in the range of $0-5 \%$ deviation from the optimal solution while a few solutions are in the range of equal to or more than $20 \%$. The average percentage (only for non-optimal cases) is about 7.9384 across all cases. These results indicate that the algorithm is able to generate high quality solutions.

\subsection{Comparison of Running Times}

Table 3 contains the average running times of the proposed algorithm for each experiment. For comparison, the time taken to generate the optimal solutions by using exhaustive search are also listed. The algorithm was implemented on a SPARC IPX workstation and the time data was measured in milli-seconds. As can be seen from the table, the times taken by the proposed algorithm are reasonable. From the experiment results presented in the previous section, we observe that there is a trade-off between execution time and solution quality.

Table 3: Average running times (msecs) of the algorithm.

\begin{tabular}{|c|c||c|c|}
\hline $\begin{array}{l}\text { No. of } \\
\text { Sites }\end{array}$ & $\begin{array}{l}\text { No. of } \\
M D O \text { s }\end{array}$ & $\begin{array}{l}\text { Exhaustive } \\
\text { Search }\end{array}$ & $\begin{array}{l}\text { Hill } \\
\text { Climbing }\end{array}$ \\
\hline \hline 4 & 4 & 7.63 & 38.95 \\
\hline 4 & 8 & 6457.69 & 1014.08 \\
\hline 5 & 4 & 18.34 & 68.36 \\
\hline 5 & 8 & 50224.66 & 2011.78 \\
\hline 6 & 4 & 51.88 & 85.14 \\
\hline 6 & 8 & 273494.96 & 2885.52 \\
\hline 7 & 4 & 176.10 & 166.09 \\
\hline 7 & 8 & 1587830.28 & 4721.17 \\
\hline 8 & 4 & 333.23 & 169.28 \\
\hline 8 & 8 & 5755754.63 & 12053.53 \\
\hline
\end{tabular}

\section{Summary}

In this paper, we have develop a probabilistic navigational model for modeling the user behavior while browsing hypermedia documents. This model is used to calculate the expected number of accesses to each hypermedia document from each site. The synchronization constraints for presenting the MDOs of hypermedia documents are modeled by using the OCPN specification. A cost model is developed to calculate the average response time observed by the end-users while browsing a set of hypermedia documents for a given allocation of MDOs. This cost model is generalized to take into consider end-user interaction while accessing MDOs, and limited buffer space constraints at the end-user site. We have also proposed an MDO data allocation algorithm based on Hill-climbing heuristic. Results indicate that there is a trade-off between execution time and solution quality and the proposed algorithm is a viable choice for small problem sizes.

\section{References}

[1] P. B. Berra, C. Y. R. Chen, A. Ghafoor, C.C. Lin, T. D. C. Little, and D. Shin, "Architecture for Distributed Multimedia Systems", Computer Communications vol.13, no.4 (May 1990) p217-31.

[2] R. Erfle, "HyTime as the multimedia document model of choice," Proc. of the Int. Conf. on Multimedia Computing and Systems, Cat. No. 94TH0631-2, 1994, pp.445-454.
[3] K. P. Eswaran, "Placement of Records in a File and File Allocation in a Computer Network," Information Processing, 1974, pp.304-307.

[4] A. Ghafoor, "Multimedia database management systems," ACM Computing Surveys, 27(4) Dec. 1995, pp.593-598.

[5] C.F. Goldfarb, "Standards-HyTime: a standard for structured hypermedia interchange," IEEE Computer, 24(8), 1991, 81-84.

[6] F. Kappe, G. Pani and F. Schnabel, "The architecture of a massively distributed hypermedia system," Internet Research, vol. 3, no. 1, Spring 1993, pp.10-24.

[7] Y. Kwok, K. Karlapalem, I. Ahmad, and N. M. Pun, "Design and Evaluation of Data Allocation Algorithms for Distributed Multimedia Database Systems," IEEE JSAC, 14(7) 1996, pp. 1332-1348.

[8] T. D. C. Little, "Synchronization and storage models for multimedia objects," IEEE JSAC, 8(3) April 1990, pp.413427.

[9] S. R. Newcomb, "Multimedia interchange using SGML/ HyTime," IEEE Multimedia, 2(2) 1995, pp. 86-89.

[10] B. Prabhakaran and S. V. Raghavan, "Synchronization models for multimedia presentation with user participation," Multimedia Systems, 1994, vol. 2, pp.53-62.

[11] J. Song, Y. N. Doganata, M. Y. Kim and A. N. Tantawi, "Modeling Timed User-Interactions in Multimedia Documents," Proc. Int. Conf. on Multimedia Computing and Systems, 1996, pp.407-416.

[12] P. D. Stotts and R. Furuta, "Petri-net-based hypertext: document structure with browsing semantics," ACM TOIS, 7(1) 1989, pp.3-29.

[13] H. M. Taylor and S. Karlin, An Introduction to stochastic modeling, Academic Press, 1994.

[14] S. Vuong, K. Cooper and M. Ito, "Specification of Synchronization Requirements for Distributed Multimedia Systems," Proc. Int.l Workshop on Multimedia Software Development, 1996, pp.110-119.

[15] M. Woo, N.U. Qazi, and A. Ghafoor, “A Synchronization Framework for Communication of Pre-orchestrated Multimedia Information," IEEE Network, pp. 52-61, January/February 1994. 